\section{Anatomical Determinants of Facial Identity: The Central Importance of Retaining Ligaments and SMAS}

\section{Abstract}

Background: Facial rejuvenation constitutes only one aspect of facelift-surgery. Another, often underestimated central challenge of facelift-surgery is to maintain the patient's facial-identity. Therefore it is important to identify the main anatomical determinants of facial-identity. Their recognition impacts on designing optimal facelift-surgery techniques.

Objective: To identify which structures in human facial anatomy, that are accessible to facelift surgeons, play a central role in determining a patient's personal facialidentify.

Methods: First, the master anatomical constituents of human facial anatomy are reconsidered. Second, it is evaluated which of these constituents are important for identity-determining facial expression. Third, from frequent undesired results of well-defined standard facelift-surgery techniques it is concluded whether or not dissection of a given anatomical constituent is important for conserving facialidentify.

Results: On this basis, we argue that retaining ligaments and the inter segmental connections of the Superficial-Musculo-Aponeurotic-System (SMAS), which tether, structure and compartmentalize facial soft tissue, are the key anatomical determinants of human facial-identity. Conclusion: Optimal facelift-surgery should strive for conserving a patient's facial-identity. In order to achieve this, retaining ligaments should be preserved and therefore the superficial-musculo-aponeurotic-system (SMAS) will be only dissected related to the tissue-layer main interconnections keeping the retaining ligaments and the related layers connected during surgery.

Keywords: Facial rejuvenation; Superficial-musculo-aponeurotic-system (SMAS); Face-lifting
Funk $\mathbf{W}^{1}$ and

\section{Hans-Robert Metelmann²}

1 Clinic for Aesthetic, Cosmetic and Reconstructive Surgery, Munchen, Germany

2 The Clinic for Oral Jaw Facial Surgery/ Plastic Surgery, University of Greifswald, 17475 Greifswald, Germany

Corresponding author: Funk W

ほ info@schoenheitsklinik.com

Clinic for Aesthetic, Cosmetic and Reconstructive Surgery, Ms Holle-Strasse 32, 81739 Munchen, Germany.

Tel: $+49(0) 89 / 6060900$

Fax: $+49(0) 89 / 6061604$

Citation: Funk W, Metelmann HR. Anatomical Determinants of Facial Identity: The Central Importance of Retaining Ligaments and SMAS. J Aesthet Reconstr Surg. 2017, 3:1.

Received: December 30, 2016; Accepted: February 23, 2017; Published: March 02, 2017

\section{Introduction}

While facial rejuvenation evidently is a key target of facelifting, another-equally important, yet often underestimatedcentral challenge of facelift-surgery is to maintain a patient's facial-identity. Here we propose for the first time that retaining ligaments and the Superficial-Musculo-Aponeurotic-System (SMAS) operate as "guardians of facial-identity", since they control facial dynamics and fix facial statics. Therefore, the preservation of these structures during facelift-surgery is elementary to the conservation of human facial-identify $[1,2]$.
Facial-identity is a key component of individuality and encompasses the totality of visual signals sent by a human individual's face. Identity results from differences in the appearance of defined facial regions (e.g., eyebrow/eye/nose/ cheek/chin/mouth positioning and form, forehead shape), which objectively distinguish an individual's face from that of others in a quantifiable manner [3]. Besides eye- and mouth-related signals these include facial soft tissue arrangement and proportions 
that are fixed by underlying hard, soft, and tensile structures (e.g., facial bones, subcutis, malar and Bichat fat body, muscle tendons, retaining ligaments, SMAS). In this system, muscles and tendons arrange the dynamics of facial expressions, while retaining ligaments and SMAS organize facial statics.

Gender, ethnic origin, emotions, social status, general health and multiple other information are transported by the individual proportions and expressions of a given human's face and form an indispensable social interaction tool for this individual. Facialidentity is intimately interwoven with face perception, i.e., the complex neurobiological processes by which the central nervous system recognizes and interprets the human face [2]. Therefore, preservation of a recognizable facial-identity throughout life is the best guarantee of relatively constant facial perception by others. This, in turn, stabilizes an individual's self-perception and personality.

For these reasons, it is important to identify the main anatomical determinants of facial-identity when designing optimal faceliftsurgery techniques. Here, we attempt to do so by adopting the simple method described below.

\section{Methods}

First, the master constituents of human facial anatomy are reconsidered. Second, it is evaluated which of these constituents are instrumental for the maintenance of identity-determining facial expression. Third, from frequent undesired results of welldefined standard facelift-surgery techniques it is concluded whether or not the dissection of a given anatomical constituent is important for conserving facial-identify.

\section{Results}

Facial expression is governed by the interplay of static, volumegenerating, and dynamic elements. Osseous structures provide the central scaffold of a human face and its master static element. However, other important (semi-)static elements of facial anatomy are tendons, retaining ligaments, the SMAS, fat and connective tissue (Figure 1). The master volume-generating elements of the face are the subcutaneous, compartmentalized fat system and the deep fat bodies (e.g. malar, SOOF, Bichat) (Figure 2), while facial muscles bestow facial dynamics. The osseous scaffold is directly or indirectly linked by the periosteum to the entire rest of the facial tissue mass. The latter is highly compartmentalized. Each compartment consists of muscle, fat, connective tissue, tendons, ligaments, blood vessels, nerves and the overlying skin. Among these anatomical constituents, the volume-generating fat compartments have the most profound impact on facial expression (and thereby on facial-identity). However, this impact is greatly shaped by the fixation of fat in a given facial compartment by the retaining ligaments and by the SMAS, which tether, structure and compartmentalize facial soft tissue (Figure 3). Retaining ligaments and SMAS operate as static fixations and at the same time limit muscle activity-based facial dynamics $[4,5]$. Therefore, both structures are top candidates as master determinants of volume-independent facial expression. To critically explore whether retaining ligaments and SMAS really

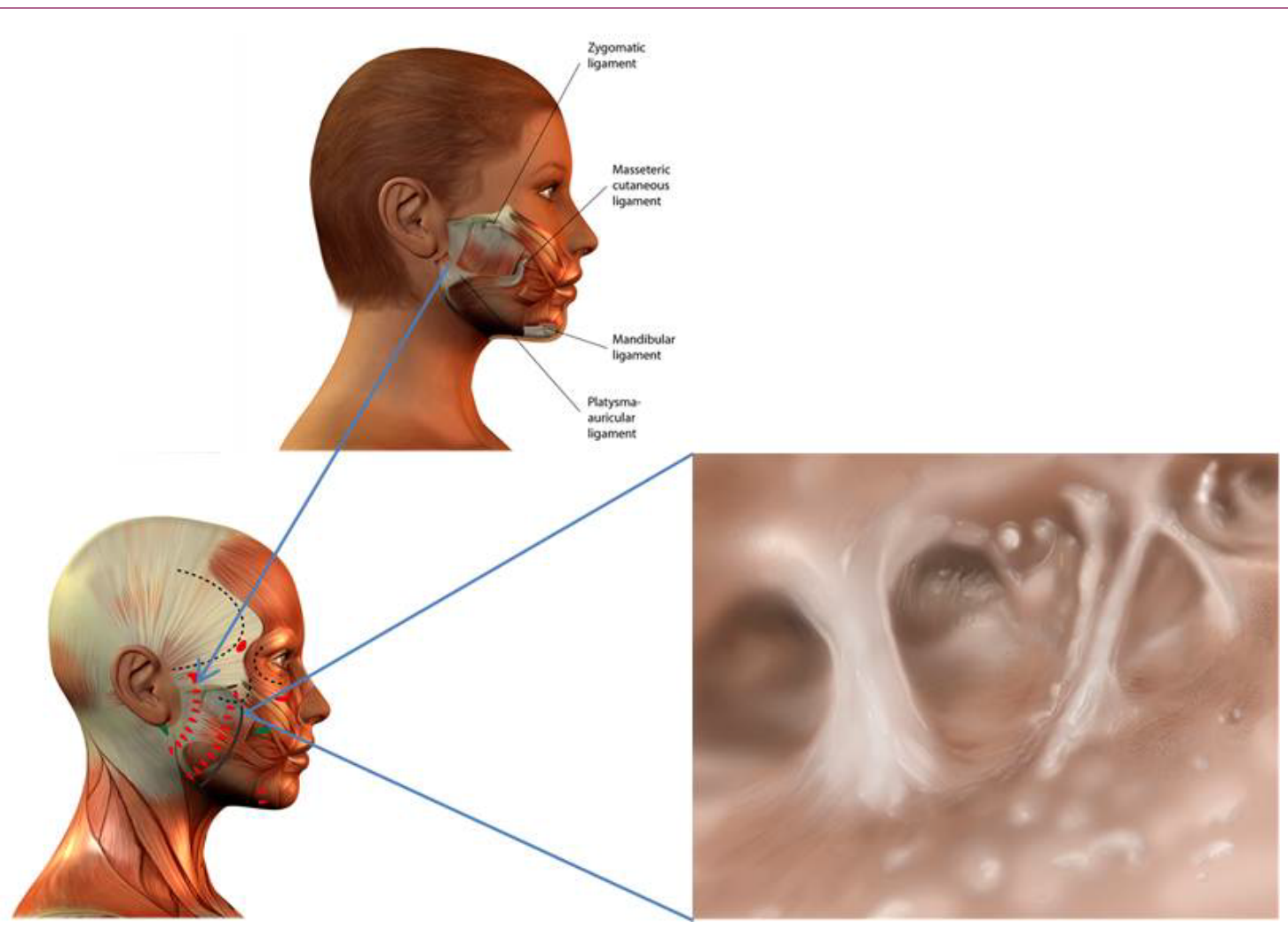

Figure 1 Retaining ligaments. 

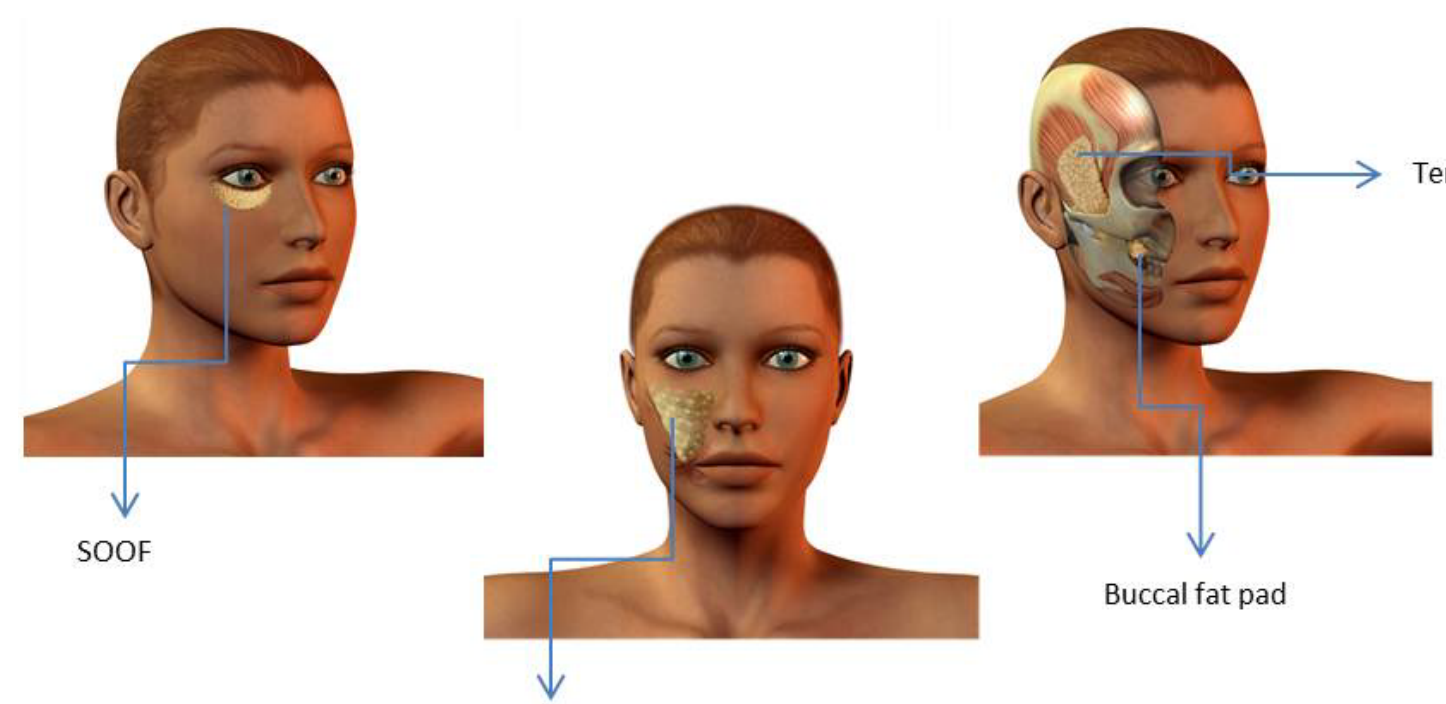

Buccal fat pad

Malar fat pad

Figure 2 Facial fat pad distribution.

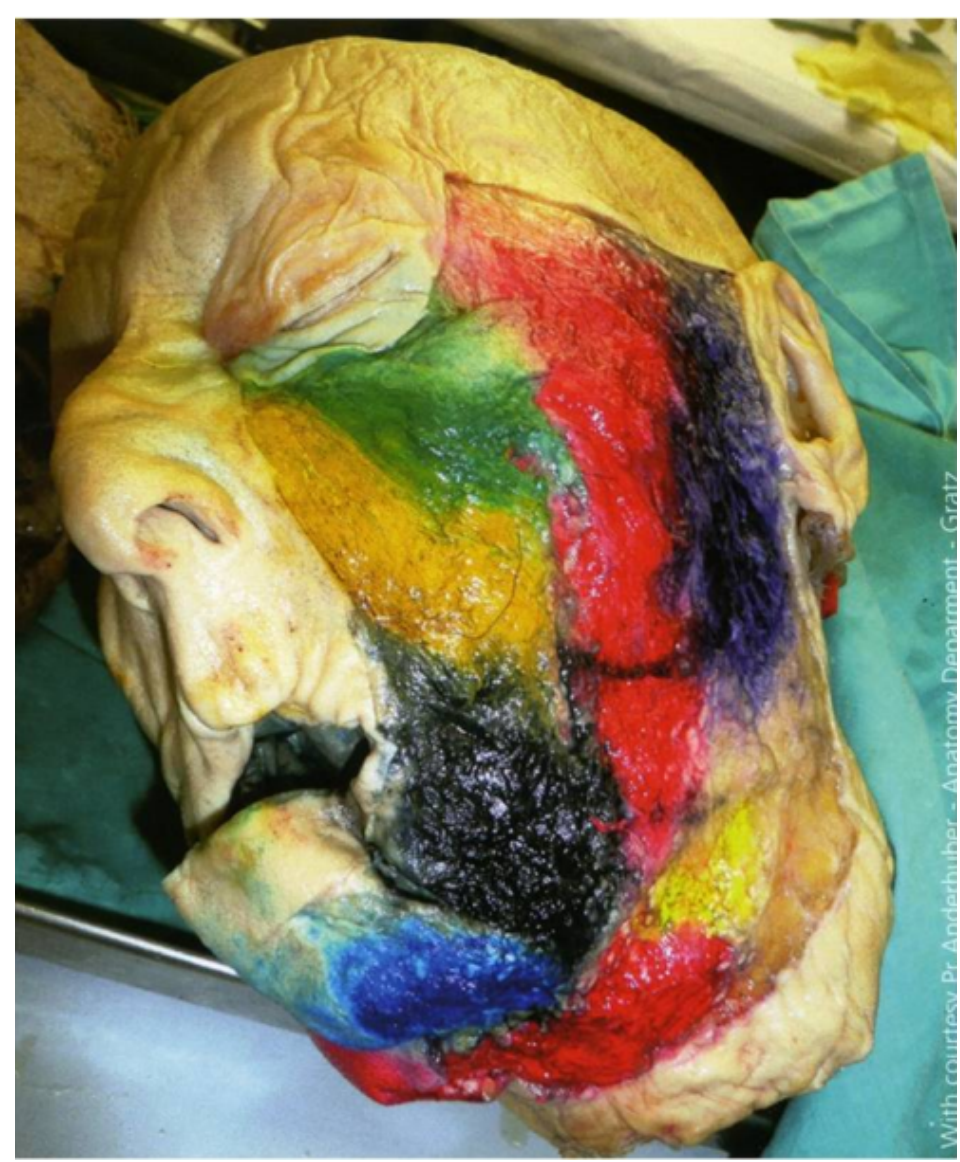

Figure 3 Distribution of the different compartments.

execute this proposed function, it suffices to examine the facial expression consequences of their dissection during conventional facelift-surgery. These consequences are readily seen during SMAS dissection and opening of the retaining ligaments according to Stuzin [5]: Overextension and hyper volumizing of the cheekbones, and unnatural, mask-like forming of the perioral and periorbital area (Figure 4). Therefore, the aesthetic outcome of SMAS dissection is well-recognized to be highly dependent 


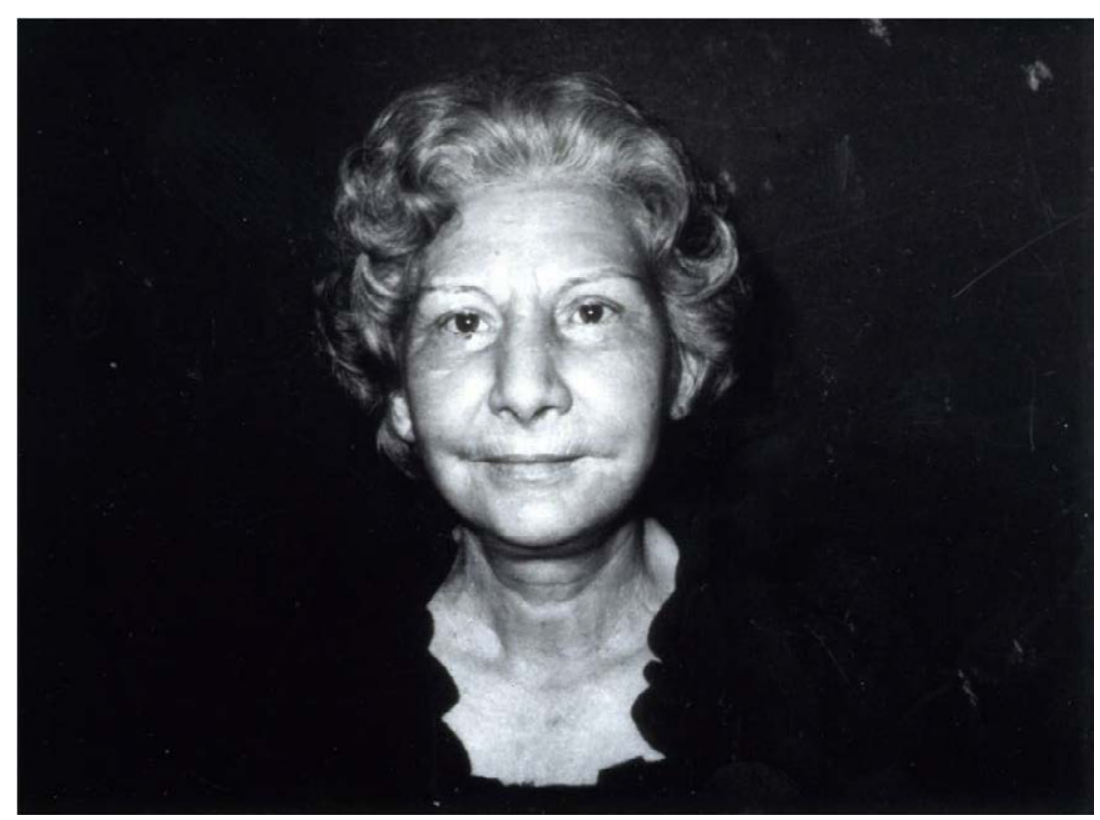

Figure 4 Overextension and hyper volumizing of the cheekbones.

on how well the severed retaining ligaments and the SMAS are being refixed during facelift-surgery [5]. On this basis, retaining ligaments and the intersegmental connections of the SMAS, are the key anatomical determinants of human facial identity. Dissection of these structures during facelift-surgery, therefore, endangers the conservation of facial-identity as defined above, if refixation of these structures remains insufficient/unsatisfactory.

\section{Conclusion}

In order to optimally conserve a patient's facial-identity during facelift surgery, retaining ligaments and the SMAS should therefore be preserved, since they serve as "guardians of facialidentity". On this theoretical basis, we have recently reported a novel, rapidly executed face-lifting technique that is based on the preservation of retaining ligaments and SMAS tethering
(PRESTO) (for procedural details, see Funk et al, submitted). The clinical success of this technique underscores the importance of retaining ligaments and SMAS in facial-identity.

\section{Acknowledgement}

The authors express their gratitude to the surgeons and stuff of the division of Maxillo-Facial-Surgery and Plastic Surgery at the University of Greifswald and the physicians from the Anatomical Institute from the University of Homburg-Saar and the Anatomical Institute of the Medical University of Graz.

\section{Conflict of Interest}

The authors have no financial disclosures, commercial associations, or conflicts of interest to report. 


\section{References}

1 Brandt MG, Hassa A, Roth K, Wehril B, Moore CC (2012) Biomechanical Properties of the Facial Retaining Ligaments. Arch Facial Plast Surg.

2 Calder AJ, Young AW (2005) Understanding the recognition of facial identity and facial expression. Nat Rev Neurosci 6: 641-651.
3 Kau CH (2011) Creation of the virtual patient for the study of facial morphology. Facial Plast Surg Clin North Am 19: 615-622.

4 Rohrich RJ, Pessa JE (2008) The retaining system of the face: histologic evaluation of the septal boundaries of the subcutaneous fat compartments. Plast Reconstr Surg 121: 1804-1809.

5 Stuzin JM, Baker TJ, Gordon HL (1992) The relationship of the superficial and deep facial fascias: relevance to rhytidectomy and aging. Plast Reconstr Surg 89: 441-449. 